Agrotrópica 32(2): 97 - 104. 2020.

Centro de Pesquisas do Cacau, Ilhéus, Bahia, Brasil

\title{
DESEMPENHO DE ESPÉCIES MADEIREIRAS EM SISTEMA AGROFLORESTAL DE CACAUEIRO E SERINGUEIRA NO SUL DA BAHIA, BRASIL
}

\author{
Daniel Piotto $^{1 *}$, José Raimundo Bonadie Marques ${ }^{2}$, Andrei Caíque Pires Nunes ${ }^{1}$ \\ ${ }^{1}$ Universidade Federal do Sul da Bahia, Centro de Formação em Ciências Agroflorestais, 45613-204, Itabuna, BA, Brasil. \\ daniel.piotto@ufsb.edu.br; andrei.nunes@ufsb.edu.br. \\ ${ }^{2}$ Comissão Executiva do Plano da Lavoura Cacaueira (CEPLAC/ CEPEC), 45604-811, Ilhéus, BA, Brasil. \\ bonadiemarques@yahoo.com.br \\ *Autor para correspondência: daniel.piotto@ufsb.edu.br
}

\begin{abstract}
Mesmo havendo grandes áreas ocupadas por sistemas agroflorestais (SAFs) nos trópicos, pouco ainda é aproveitado do potencial de produção de madeira sólida de alta qualidade dessas áreas. Neste estudo, foi testado o efeito de três diferentes densidades de plantio de espécies madeireiras consorciadas com seringueira e cacaueiro sobre o crescimento e a produtividade de Cordia alliodora, Tectona grandis e Swietenia macrophylla, que representam essências florestais madeireiras com valor econômico promissoras na região Sul da Bahia. Na idade de 12 anos, todos os indivíduos de espécies madeireiras foram medidos e classificados quanto a forma do fuste. Os resultados indicaram que a densidade de indivíduos de árvores madeireiras não teve efeitos no crescimento e na produtividade das árvores. Porém, o crescimento e a produtividade foram significativamente diferentes entre as espécies madeireiras estudadas. C. alliodora teve um crescimento superior às outras espécies. $T$. grandis e $S$. macrophylla tiveram taxas de crescimento similares. C. alliodora foi a espécie mais produtiva, mostrando diferenças significativas em volume e alcançando o dobro da produtividade encontrada quando comparada as outras espécies. Os resultados indicam um grande potencial dos SAFs para produção de madeira na região Sul da Bahia.
\end{abstract}

Palavras-chave: crescimento, produtividade, Cordia alliodora, Tectona grandis, Swietenia macrophylla, SAF.

\footnotetext{
Performance of timber species in agroforestry system with cocoa and rubber trees in southern Bahia, Brazil. Besides the large areas occupied by agroforestry systems in the tropics, little is yet known about the potential for producing high quality timber in these areas. In this study, we tested the effect of three different planting densities of timber species mixed with rubber and cocoa agroforestry system on the growth and yield of Cordia alliodora, Tectona grandis and Swietenia macrophylla, which represent promising timber species for economic uses in southern Bahia. At the age of 12 years, all individuals of timber species were measured and classified for stem shape. The results indicated that the density of timber species had no effect on growth and yield. However, growth and yield were significantly different among the species studied. $C$. alliodora had a significantly higher growth than the other species. T. grandis and S. macrophylla had similar growth rates. C. alliodora was the most productive species, showing significant differences in volume when compared to other species and reaching twice of the yield found for T. grandis and S. macrophylla. The results of this study indicate a great potential for timber production in agroforestry systems in southern Bahia.
}

Key words: growth, yield, Cordia alliodora, Tectona grandis, Swietenia macrophylla 


\section{Introdução}

Existem muitas espécies de árvores nativas do Brasil e exóticas que são utilizadas pela indústria florestal nos trópicos. A indústria florestal brasileira possui relevância para o setor econômico do país, contribuindo para saldos favoráveis na balança comercial, incremento de PIB e geração de cerca de 508 mil empregos diretos, vinculados a produção de diversos produtos madeireiros e não madeireiros (IBA, 2016). Apesar desse impacto econômico considerável do setor florestal, o uso de sistemas agroflorestais (SAFs) e plantações mistas para suprimento de madeira não tem sido explorado adequadamente nos últimos anos sob o ponto de vista econômico. Praticamente, a totalidade da produção de madeira atual é proveniente de florestas naturais ou reflorestamentos desenhados exclusivamente para produção de madeira sólida (Evans \& Turnbull, 2004; FAO, 2015).

Apesar de haverem grandes superfícies ocupadas por SAFs nos trópicos, pouco ainda é aproveitado do potencial de produção de madeira sólida de alta qualidade nessas áreas, bem como do potencial de provisão de serviços ecossistêmicos (Montagnini, 2017). Tradicionalmente, o componente arbóreo de SAFs sempre foi manejado para favorecer os outros cultivos associados, provendo sombra, conforto térmico e microclima, ciclagem de nutrientes, entre outros serviços que visam garantir a produtividade dos cultivos agrícolas (Ashton \& Montagnini, 2000; Silva, 2013). Porém, com a crescente demanda de produtos florestais e a crítica situação das reservas madeireiras em florestas naturais, existe uma necessidade urgente de fomentar programas de reflorestamento em larga escala para atender a demanda atual e futura de madeira sólida, incluindo a produção madeireira em associação com outros cultivos na forma de sistemas agroflorestais (FAO, 2015).

Apesar de haverem mais de um bilhão de hectares destinados a SAFs no planeta, representando mais de um terço da área agrícola global (Zomer et al., 2016), ainda são incipientes os estudos para quantificação do potencial de crescimento e produtividade de essências florestais madeireiras em SAFs, mesmo em regiões onde são amplamente utilizados como a Mata Atlântica e Amazônia (May e Trovatto, 2008).
No Sul da Bahia, a Mata Atlântica foi fortemente impactada pela ação antrópica, alcançando picos de desmatamento e degradação no início da década de 1990 e atualmente conta apenas $11,1 \%$ da área de floresta original (SOS Mata Atlântica, 2019). Com a rápida degradação florestal, boa parte dos produtos florestais em madeira sólida disponíveis no mercado da região começou a ser importado da Amazônia e de outras regiões do país. Com isso, apesar da região Sul da Bahia representar um dos maiores celeiros agroflorestais do planeta, com mais de 400.000 hectares de cacau cultivados em sistemas agroflorestais (Leite, 2018), pouco vem sendo explorado do grande potencial dos SAFs da região para produção de produtos florestais madeireiros de alta qualidade e geração de serviços ecossistêmicos, como o sequestro de carbono.

Recentemente, vários produtores rurais e agências de fomento e crédito têm investido no estabelecimento de SAFs que incluem árvores de rápido crescimento para fins madeireiros e com alto valor comercial. Porém, os produtores rurais, empresas privadas e investidores necessitam de uma base de informação mais sólida sobre o desempenho das principais essências florestais, tanto para construção de planos de negócio, como para o desenvolvimento de cenários de produção em médio e longo prazo. Nesse sentido, a geração de informações sobre o crescimento e a produtividade de essências florestais em SAFs é essencial para promover a inclusão de espécies florestais madeireiras nesses sistemas e aumentar a renda e oferta de madeira plantada no mercado de produtos florestais.

Neste estudo, foi testado o efeito de três diferentes densidades de plantio de espécies madeireiras em SAF com seringueira, cacaueiro e bananeira sobre o crescimento e a produtividade de Cordia alliodora, Tectona grandis e Swietenia macrophylla, que representam essências florestais promissoras para região Sul da Bahia. Os resultados de crescimento e produtividade das espécies florestais madeireiras estudadas em SAFs foram comparados com dados de crescimento das espécies em reflorestamento homogêneo, com intuito de ilustrar o potencial de produção de madeira sólida em sistemas agroflorestais. 


\section{Material e Métodos}

O SAF foi estabelecido em uma área experimental do Centro de Pesquisas do Cacau (CEPEC), em que o cacaueiro foi implantado sob a sombra permanente de eritrinas (Erythrina spp.) no sistema conhecido regionalmente como derruba total (Marques e Monteiro, 2016), na Quadra E, situada na sede da Comissão Executiva do Plano da Lavoura Cacaueira (CEPLAC), no município de Ilhéus, Bahia. A área experimental está inserida na região onde tradicionalmente são cultivados os cacaueiros, com altitude de 51 metros sobre o nível do mar e compreendida entre a latitude de $14^{\circ} 45^{\prime} \mathrm{S}$ e longitude de $39^{\circ} 13^{\prime}$ W. Segundo a classificação de Köppen, este clima se caracteriza como do tipo Am, com precipitação e temperatura médias anuais de $1700 \mathrm{~mm}$ e $23,5^{\circ} \mathrm{C}$, respectivamente, e a média anual de umidade relativa do ar é de 85,3\% (Almeida, 2001). O solo é classificado como do tipo Cepec, com textura média, de alta fertilidade, relevo plano, porém mal drenado (Santana et al., 2002; Faria Filho e Araújo, 2003).

Após a eliminação total das eritrinas, foi realizado o preparo da área, balizamento e abertura de covas para o plantio das seringueiras, bananeiras, milho e as essências florestais. Os cacaueiros remanescentes na área foram enxertados em brotos basais e a recomposição do estande foi realizada com o plantio de mudas enxertadas preparadas em casa de vegetação (Marques e Monteiro, 2016). As covas para os cultivos perenes foram abertas nas dimensões de $0,50 \mathrm{~m} \times$ $0,50 \mathrm{~m} \times 0,60 \mathrm{~m}$, aplicando-se $200 \mathrm{~g}$ de calcário dolomítico como corretivo e fonte de cálcio e magnésio. No ato do plantio, as plantas foram adubadas com 170 g de superfosfato simples em mistura com a camada superior do solo. O plantio das culturas temporárias foi realizado logo após o preparo do terreno. A bananeira (Musa spp.) foi plantada no espaçamento de 3,0 $\mathrm{m} \times$ 3,0 m, enquanto o milho (Zea mays), foi cultivado por dois ciclos consecutivos, sem obedecer a um espaçamento definido. O cacaueiro e a seringueira foram estabelecidos em uma densidade de 1.111 plantas/ha e 333 plantas/ha, respectivamente.

O plantio das espécies madeireiras foi realizado no ano de 2005, um ano após o plantio da seringueira. Foram selecionadas três espécies florestais madeireiras: Cordia alliodora (claraíba ou louro- freijó), Tectona grandis (teca), e Swietenia macrophylla (mogno brasileiro). C. alliodora é uma espécie com ampla distribuição nos trópicos americanos, ocorrendo desde o México até o Brasil, e conhecida pelo seu alto valor para uso madeireiro (Carvalho, 2007). T. grandis é uma espécie nativa do sudeste asiático que vem sendo bastante estudada e amplamente cultivada em regiões tropicais para produção de madeira sólida (Ugalde, 2013). S. macrophylla é uma espécie nativa da Amazônia que, apesar de estar na lista vermelha de espécies ameaçadas, apresenta madeira de alto valor comercial e grande potencial silvicultural (Mayhew \& Newton, 1998).

As mudas foram plantadas intercaladas nas mesmas fileiras da seringueira, com uma densidade de plantas por hectare de 130 no talhão 1,117 no talhão 2 e 90 no talhão 3. Todos os indivíduos de espécies madeireiras foram medidos no ano de 2017. Cada indivíduo teve o diâmetro à altura do peito (DAP) medido com fita diamétrica, altura comercial (m) e altura total (m) medidos com Nikkon Forestry Pro laser rangefinder. Também foram coletados dados de forma do fuste, classificados como classe 1 (reto) ou classe 2 (torto e/ou bifurcado).

Todos dados da medição realizada em 2017 para as variáveis DAP, altura e forma foram utilizados para o cálculo de variáveis de crescimento e produtividade. As variáveis calculadas foram: DAP médio, altura total média, altura comercial média e volume total, bem como o incremento médio anual (IMA) em DAP e em volume por hectare para a idade de 12 anos. Para o cálculo do volume total foi utilizado um fator de forma de 0,5 (Newbould, 1967).

Para a análise de dados foi considerado o modelo de um delineamento inteiramente casualizado, conforme descrito a seguir:

$$
\begin{aligned}
& \text { Yijk = m + Gi }+A j+G A i j+E i j k \\
& \text { Onde: } \mathrm{i}=\text { espécies; } \\
& \mathrm{j}=\text { talhões; } \\
& \mathrm{k}=\text { indivíduos }
\end{aligned}
$$

Os dados foram processados no software $\mathrm{R}(\mathrm{R}$ Core Team, 2020) e comparados entre talhões e entre as espécies para determinar as melhores densidades de plantio e as espécies mais promissoras para região. Foi considerado um arranjo fatorial dentro do 
delineamento inteiramente casualizado, onde um fator representou os talhões (diferentes densidades) e o outro fator representou as espécies (C. alliodora, $S$. macrophylla e $T$. grandis), além de se considerar a interação entre os dois fatores.

Foi realizado o teste de normalidade dos dados (Shapiro \& Wilk, 1965) e de homogeneidade de variâncias de Bartlett (1937). Para as variáveis com distribuição normal (DAP, altura total, altura comercial, volume total por indivíduo, IMA em DAP, e IMA em volume) foi realizada uma análise de variância (teste F a $8 \%$ de significância) e teste de agrupamento de médias pelo teste de Scott-Knott (1974), em nível de $5 \%$ de significância. Para as variáveis que não apresentaram distribuição normal (forma e sanidade), foi realizada uma análise de Kruskal-Wallis para verificação de diferenças entre os níveis de cada fonte de variação e a análise de comparações múltiplas de Nemenyi.

As médias obtidas para cada espécie também foram comparadas com dados da literatura sobre reflorestamentos homogêneos de C. alliodora, $T$. grandis e $S$. macrophylla. Para comparação foram utilizados incrementos anuais para as diferentes variáveis estudadas (variável/idade).

\section{Resultados e Discussão}

Os resultados da análise de variância indicaram que a densidade de indivíduos de árvores madeireiras não teve efeitos significativos nas variáveis de crescimento e de produtividade das três espécies madeireiras estudadas (Tabela 1). Contudo, foi detectado efeito da interação entre a densidade de indivíduos e as espécies testadas, indicando que as espécies responderam diferentemente a densidade de indivíduos. Enquanto C. alliodora mostrou maior produtividade no talhão com menor densidade de indivíduos, $T$. grandis obteve maior crescimento em diâmetro no talhão com maior densidade de indivíduos (Tabela 2).

$\mathrm{O}$ crescimento e a produtividade foram significativamente diferentes entre as espécies estudadas (Tabela 1). Os resultados das medições tomadas aos 12 anos de idade mostraram que $C$. alliodora teve um crescimento em altura total e comercial significativamente superior as outras espécies. $T$. grandis e $S$. macrophylla tiveram médias de crescimento em altura similares. O crescimento em diâmetro de C. alliodora foi o mais expressivo, significativamente superior ao encontrado para $T$. grandis e S. macrophylla. S. macrophylla obteve os menores valores médios de DAP aos 12 anos (Tabela 3). No geral, o incremento médio anual em DAP para todas as espécies foi extremamente alto, com valores superiores aos $2 \mathrm{~cm} / \mathrm{ano}$, sendo que $C$. alliodora alcançou um IMA DAP próximo dos $4 \mathrm{~cm} /$ ano (Tabela 3).

As árvores de $C$. alliodora foram as mais produtivas, mostrando diferenças significativas em volume quando comparadas as outras espécies e alcançando o dobro da produtividade encontrada para T. grandis e S. macrophylla. Não foram observadas diferenças significativas entre a produção volumétrica de $T$. grandis e S. macrophylla (Tabela 3). O incremente médio anual em volume por hectare foi alto para todas as espécies estudadas, considerando que a densidade de árvores madeireiras num sistema agroflorestal é muito mais baixa quando comparado a um povoamento florestal puro. O melhor IMA Volume foi para $C$. alliodora com uma média de $9,26 \mathrm{~m}^{3} / \mathrm{ha} /$ ano e o pior foi para $S$. macrophylla com uma média de $4,08 \mathrm{~m}^{3} /$ ha/ano (Tabela 3 ).

Com relação a forma do fuste, os indivíduos de $T$. grandis apresentaram forma mais retilínea, quando comparados as outras espécies estudadas. No geral, os indivíduos de $C$. alliodora também apresentaram uma excelente forma, porém alguns indivíduos apresentaram tortuosidade na formação do fuste. Os fustes de pior forma foram das árvores de $S$. macrophylla, que apresentaram muitos problemas de forma ocasionado pelo ataque de pragas de ponteiro (Hypsipyla grandella Zeller), gerando fustes curtos e bifurcados (Tabela 4). Também foi detectado efeito da interação entre a densidade de indivíduos e a forma das espécies testadas, com $T$. grandis apresentando melhor forma em talhões com menor densidade de árvores (Tabela 5).

Diversos ensaios com essências florestais nativas e exóticas foram estabelecidos na região Sul da Bahia pela CEPLAC há décadas (Da Vinha e Lobão, 1989). Essas áreas experimentais possibilitaram o estudo do potencial de mais de 200 essências nativas e exóticas no longo prazo. Entre as espécies estudadas estavam C. alliodora, T. grandis e S. macrophylla, que não 
Tabela 1 - Análise de variância para as variáveis com distribuição normal (DAP, altura total, altura comercial, volume total, IMA em DAP, e IMA em volume) e análise de Kruskal-Wallis para as variáveis forma e sanidade, para verificação de diferenças entre níveis dos fatores em estudo relativo ao desempenho de essências florestais madeireiras em sistemas agroflorestais com 12 anos de idade, estabelecido no CEPEC, Bahia, Brasil

\begin{tabular}{lcccc}
\hline & DAP & Altura total & Altura comercial & Forma \\
\cline { 2 - 5 } & p-valor & p-valor & p-valor & p-valor \\
\hline Talhão & 0,279 & 0,109 & 0,102 & 0,091 \\
Espécie & $<0,001^{*}$ & $<0,001^{*}$ & $<0,001^{*}$ & $<0,001^{*}$ \\
Talhão x Espécie & $0,011^{*}$ & 0,447 & 0,210 & $<0,001^{*}$ \\
CV(\%) & 17,600 & 14,053 & 28,444 & - \\
\hline & Sanidade & IMAdap & Volume total & IMAvolume \\
\cline { 2 - 5 } & p-valor & p-valor & p-valor & p-valor \\
\hline Talhão & 0,697 & 0,279 & 0,536 & 0,536 \\
Espécie & 0,634 & $<0,001^{*}$ & $<0,001^{*}$ & $<0,001^{*}$ \\
Talhão x Espécie & 0,962 & $0,005^{*}$ & $0,079^{*}$ & $0,079^{*}$ \\
CV(\%) & - & 17,600 & 43,712 & 43,712 \\
\hline
\end{tabular}

* significativo à $8 \%$ de probabilidade.

Tabela 2 - Desdobramento das interações significativas por meio do teste de Scott-Knott em nível de 5\% de probabilidade para variáveis dendrométricas com distribuição normal de essências florestais madeireiras em sistemas agroflorestais com 12 anos de idade, estabelecido no CEPEC, Bahia, Brasil. As médias seguidas por letras diferentes em cada variável possuem diferenças significativas

\begin{tabular}{lcccccc}
\hline \multirow{2}{*}{ Espécie } & \multicolumn{3}{c}{ DAP $(\mathbf{c m})$} & \multicolumn{3}{c}{ IMAdap (cm/ano) } \\
\cline { 2 - 7 } & $\mathrm{T} 1$ & $\mathrm{~T} 2$ & $\mathrm{~T} 3$ & $\mathrm{~T} 1$ & $\mathrm{~T} 2$ & $\mathrm{~T} 3$ \\
\hline C.alliodora & $41,20 \mathrm{a}$ & $42,82 \mathrm{a}$ & $45,49 \mathrm{a}$ & $3,43 \mathrm{a}$ & $3,57 \mathrm{a}$ & $3,79 \mathrm{a}$ \\
S.macrophylla & $33,98 \mathrm{c}$ & $37,53 \mathrm{~b}$ & $26,13 \mathrm{~d}$ & $2,83 \mathrm{c}$ & $3,13 \mathrm{~b}$ & $2,18 \mathrm{~d}$ \\
T.grandis & $37,19 \mathrm{~b}$ & $32,68 \mathrm{c}$ & $34,63 \mathrm{c}$ & $3,10 \mathrm{~b}$ & $2,72 \mathrm{c}$ & $2,89 \mathrm{c}$ \\
\hline \multirow{2}{*}{ Espécie } & \multicolumn{2}{c}{ Volume total $\left(\mathbf{m}^{3} /\right.$ indivíduo $)$} & IMAvolume $\left(\mathbf{m}^{3} / \mathbf{h a} / \mathbf{a n o}\right)$ \\
\cline { 2 - 7 } & $\mathrm{T} 1$ & $\mathrm{~T} 2$ & $\mathrm{~T} 3$ & $\mathrm{~T} 1$ & $\mathrm{~T} 2$ & $\mathrm{~T} 3$ \\
\hline C.alliodora & $1,52 \mathrm{~b}$ & $1,74 \mathrm{a}$ & $1,86 \mathrm{a}$ & $8,42 \mathrm{~b}$ & $9,68 \mathrm{a}$ & $10,34 \mathrm{a}$ \\
S.macrophylla & $0,80 \mathrm{c}$ & $1,15 \mathrm{c}$ & $0,48 \mathrm{~d}$ & $4,42 \mathrm{c}$ & $6,37 \mathrm{c}$ & $2,69 \mathrm{~d}$ \\
T.grandis & $0,95 \mathrm{c}$ & $0,75 \mathrm{c}$ & $0,85 \mathrm{c}$ & $5,29 \mathrm{c}$ & $4,19 \mathrm{c}$ & $4,73 \mathrm{c}$ \\
\hline
\end{tabular}

Tabela 3 - Teste de agrupamento de médias pelo teste de Scott-Knott em nível de 5\% de probabilidade para variáveis dendrométricas com distribuição normal de essências florestais madeireiras em sistemas agroflorestais com 12 anos de idade, estabelecido no CEPEC, Bahia, Brasil. As espécies seguidas por letras diferentes na mesma coluna possuem médias com diferenças significativas

\begin{tabular}{|c|c|c|c|c|c|c|}
\hline \multirow[b]{2}{*}{ Espécie } & \multicolumn{2}{|c|}{$\begin{array}{l}\text { DAP } \\
(\mathrm{cm})\end{array}$} & \multicolumn{2}{|c|}{$\begin{array}{c}\text { Altura total } \\
(\mathbf{m})\end{array}$} & \multicolumn{2}{|c|}{$\begin{array}{c}\text { Altura comercial } \\
(\mathbf{m})\end{array}$} \\
\hline & Médias & $\mathrm{SK}(5 \%)$ & Médias & $\mathrm{SK}(5 \%)$ & Médias & $\operatorname{SK}(5 \%)$ \\
\hline C. alliodora & 42,67 & $\mathrm{a}$ & 22,26 & $\mathrm{a}$ & 14,35 & $\mathrm{a}$ \\
\hline T. gradis & 34,74 & $\mathrm{~b}$ & 17,32 & $\mathrm{~b}$ & 7,77 & $\mathrm{~b}$ \\
\hline \multirow[t]{2}{*}{ S. macrophylla } & 31,28 & $\mathrm{c}$ & 16,48 & $\mathrm{~b}$ & 6,23 & $\mathrm{c}$ \\
\hline & \multicolumn{2}{|c|}{$\begin{array}{l}\text { IMAdap } \\
\text { (cm/ano) }\end{array}$} & \multicolumn{2}{|c|}{$\begin{array}{c}\text { Volume total } \\
\left(\mathbf{m}^{3} / \text { individuo }\right)\end{array}$} & \multicolumn{2}{|c|}{$\begin{array}{l}\text { IMAvolume } \\
\left(\mathbf{m}^{3} / \mathbf{h a} / \text { ano }\right)\end{array}$} \\
\hline Espécie & Médias & $\mathrm{SK}(5 \%)$ & Médias & $\mathrm{SK}(5 \%)$ & Médias & $\operatorname{SK}(5 \%)$ \\
\hline C. alliodora & 3,56 & $\mathrm{a}$ & 1,667 & $\mathrm{a}$ & 9,262 & $\mathrm{a}$ \\
\hline T. gradis & 2,89 & $\mathrm{~b}$ & 0,848 & $\mathrm{~b}$ & 4,712 & $\mathrm{~b}$ \\
\hline S. macrophylla & 2,61 & $\mathrm{~b}$ & 0,735 & b & 4,084 & $\mathrm{~b}$ \\
\hline
\end{tabular}

apresentaram resultados muito promissores quando comparadas com as espécies de melhor crescimento.

Os resultados de crescimento reportados por Da Vinha e Lobão (1989) em sistemas de plantio homogêneos foram bastante diferentes dos encontrados no presente estudo em SAFs. Enquanto $S$. macrophylla obteve os melhores resultados de crescimento entre as três espécies nos ensaios da CEPLAC, com DAP médio de 14,5 $\mathrm{cm}$ aos 14 anos (Da Vinha e Lobão, 1989), no presente estudo a espécie apresentou um DAP médio de $31,3 \mathrm{~cm}$ aos 12 anos, ou seja, o crescimento em SAFs foi maior que o dobro do encontrado em povoamentos homogêneos. As diferenças de crescimento entre $T$. grandis e $C$. alliodora quando comparados os resultados de plantios homogêneos com plantios em SAFs foram ainda mais expressivos. $T$. grandis obteve resultados de crescimento pouco expressivos nos ensaios da CEPLAC, com DAP médio de 7,7 cm aos 17 anos (Da Vinha e Lobão, 1989), sendo que no presente estudo a espécie apresentou um DAP médio de $34,7 \mathrm{~cm}$ aos 12 anos. $C$. alliodora também exibiu resultados de crescimento pouco expressivos nos ensaios da CEPLAC, com DAP médio de 10,5 cm aos 15 anos (Da Vinha e Lobão, 1989), no presente estudo a espécie apresentou um DAP médio de $42,7 \mathrm{~cm}$ aos 12 anos.

Comparando os resultados de crescimento na região sul da Bahia com dados de outras regiões tropicais, observase que o crescimento de $S$. macrophylla em SAFs foi superior aos resultados esperados para monoculturas em sítios de alta qualidade nos trópicos, sendo esperado um valor de DAP médio de $22 \mathrm{~cm}$ para a idade de 12 anos (Krisnawati, Kallio e Kanninen, 2011). No caso de T. grandis, os resultados de crescimento também foram expressivos e comparáveis aos resultados de plantios homogêneos clonais no Brasil, Costa Rica e México (Ugalde, 2013). 
Para C. alliodora, os resultados de crescimento em diâmetro em SAFs no Sul da Bahia são muito superiores aos resultados de crescimento já reportados para a espécie na literatura (Carvalho, 2007; Somarriba et al., 2014). Entre as razões para o melhor desempenho de C. alliodora em SAFs pode-se elencar a alta fertilidade dos sítios onde o experimento foi estabelecido, o intensivo uso de tratos culturais como capina química, roçagem mecânica e adubação para manutenção da produtividade do cacaueiro e crescimento da seringueira, aliada a baixa densidade de árvores por hectare. Também cabe salientar que as sementes de $C$. alliodora utilizadas no experimento foram obtidas de matrizes com boa qualidade de fuste e crescimentos superiores, selecionadas no arboreto da Ceplac. Em contrapartida, as razões para o pior crescimento em diâmetro de C. alliodora em monoculturas podem ser reflexo de sítios com baixa fertilidade, falta de tratos silviculturais, variabilidade e qualidade do material genético e maior densidade de plantas por hectare.

Apesar da comparação da produtividade por hectare de SAFs e monoculturas ser enviesada pela

Tabela 4 - Análise de comparações múltiplas de Nemenyi para a variável forma de essências florestais madeireiras em sistemas agroflorestais com 12 anos de idade, estabelecido no CEPEC, Bahia, Brasil. As combinações de pares de espécies e seus respectivos valores de probabilidades indicam a igualdade ou não das mesmas

\begin{tabular}{l|cl}
\hline \multirow{2}{*}{$\begin{array}{l}\text { T. grandis } \\
\text { S. macrophylla }\end{array}$} & C. alliodora & T. grandis \\
\cline { 2 - 3 } & 0,1987 & - \\
\hline
\end{tabular}

*Significativo à $5 \%$ de probabilidade. maior densidade de árvores em monoculturas, o crescimento volumétrico significativamente superior de árvores individuais em SAFs chega a alcançar parcialmente o volume de madeira esperado para uma monocultura, bem como permite a diminuição dos ciclos de corte das espécies madeireiras. Para T. grandis e S. macrophylla, que apresentaram IMA Volume entre 4 e $5 \mathrm{~m}^{3} / \mathrm{ha} /$ ano aos 12 anos, com árvores com volume total médio entre 0,7 e $0,85 \mathrm{~m}^{3}$, já seria viável a colheita de $60 \mathrm{~m}^{3} /$ ha de madeira sólida desses SAFs. Para as mesmas espécies em monoculturas são esperadas colheitas com volumes superiores a $400 \mathrm{~m}^{3}$ por hectare em sítios de boa qualidade (Krisnawati, Kallio e Kanninen, 2011; Ugalde, 2013), porém que demandam de ciclos de corte mais longos, entre 20 e 30 anos, para produzir árvores com volumes superiores a $0,7 \mathrm{~m}^{3}$.

O crescimento excepcional de C. alliodora, com árvores com volume total médio de $1,6 \mathrm{~m}^{3}$ e IMA Volume de $9,3 \mathrm{~m}^{3} / \mathrm{ha} /$ ano aos 12 anos de idade, foram superiores ao encontrado em SAFs com cacaueiro na Costa Rica ( $5 \mathrm{~m}^{3} / \mathrm{ha} / \mathrm{ano}$ ) (Somarriba et al., 2014), bem como superiores ao encontrado em monoculturas de sua espécie congênere $C$. trichotoma em sítios com baixa fertilidade e precipitação no Espírito Santo, com IMA Volume de $6,3 \mathrm{~m}^{3} / \mathrm{ha} /$ ano aos 12 anos (Rolim e Piotto, 2018). Além da sombra e outros serviços providos pelas árvores madeireiras em SAFs, a alta produtividade concentrada em poucas árvores possibilita ciclos de corte mais curtos, melhorando as taxas de retorno de investimentos e aumentando a atratividade desses sistemas de produção.

Por outro lado, o crescimento rápido e livre de competição por luz de espécies madeireiras em SAFs

Tabela 5 - Análise de comparações múltiplas de Nemenyi para a variável forma no desdobramento da interação talhão $\times$ espécies florestais madeireiras em sistemas agroflorestais com 12 anos de idade, estabelecido no CEPEC, Bahia, Brasil. As combinações de pares de fatores e seus respectivos valores de probabilidades indicam a igualdade ou não das mesmas em nível de 5\% de significância

\begin{tabular}{|c|c|c|c|c|c|c|c|c|}
\hline & $\begin{array}{c}\mathrm{T} 1 \\
\text { C.alliodora }\end{array}$ & $\begin{array}{c}\mathrm{T} 1 \\
\text { T.grandis }\end{array}$ & $\begin{array}{c}\mathrm{T} 1 \\
\text { S.macrophylla }\end{array}$ & $\begin{array}{c}\mathrm{T} 2 \\
\text { C.alliodora }\end{array}$ & $\begin{array}{c}\mathrm{T} 2 \\
\text { T.grandis }\end{array}$ & $\begin{array}{c}\mathrm{T} 2 \\
\text { S.macrophylla }\end{array}$ & $\begin{array}{c}\mathrm{T} 3 \\
\text { C.alliodora }\end{array}$ & $\begin{array}{c}\mathrm{T} 3 \\
\text { T.grandis }\end{array}$ \\
\hline $\mathrm{T} 1-T$.grandis & 0,51 & - & - & - & - & - & - & - \\
\hline T1-S.macrophylla & 1,00 & 0,33 & - & - & - & - & - & - \\
\hline T2-C.alliodora & 0,69 & 1,00 & 0,47 & - & - & - & - & - \\
\hline $\mathrm{T} 2-T . g r a n d i s$ & 0,55 & 1,00 & 0,38 & 0,99 & - & - & - & - \\
\hline T2-S.macrophylla & 1,00 & 0,60 & 1,00 & 0,82 & 0,62 & - & - & - \\
\hline T3-C.alliodora & 1,00 & 0,93 & 0,97 & 1,00 & 0,90 & 1,00 & - & - \\
\hline T3-T.grandis & $0,01 *$ & 0,98 & $0,00 *$ & 0,40 & 1,00 & $0,02 *$ & 0,17 & - \\
\hline T3-S.macrophylla & 1,00 & 0,39 & 1,00 & 0,57 & 0,42 & 1,00 & 0,98 & $0,01 *$ \\
\hline
\end{tabular}

*Significativo à $5 \%$ de probabilidade. 
demanda cuidados especiais para formação de fustes retilíneos, não bifurcados e sem defeitos na madeira, como a presença de nós. Das três espécies estudadas, S. macrophylla foi a que apresentou maiores problemas na formação do fuste e demandou podas recorrentes de galhos para garantir uma boa forma do fuste. A má formação dos fustes de S. macrophylla ocorreu em virtude da perda precoce da dominância apical ocasionada por ataques frequentes de lagarta de mariposa, Hypsiphyla grandella Zeller, nos primeiros anos após o plantio. T. grandis e $C$. alliodora não tiveram problemas com pragas e ainda apresentaram excelente forma devido ao crescimento monopodial das espécies e manutenção da dominância apical durante todo o estudo, o que favorecerá um melhor aproveitamento da madeira na fase de processamento das árvores em serraria.

Os resultados do presente estudo indicam um grande potencial dos SAFs da região cacaueira baiana para produção de madeira sólida, porém uma das principais desvantagens dos SAFs quando comparados aos monocultivos é a colheita e aproveitamento florestal (Rolim e Piotto, 2018). Enquanto que nas monoculturas a colheita e aproveitamento florestal é uma operação simples que consiste basicamente no corte raso das árvores e arrasto dos troncos, em SAFs multifuncionais, como no caso do presente estudo, o corte e arrasto das espécies madeireiras podem causar sérios danos aos cultivos perenes consorciados, como o cacaueiro e a seringueira. Com isso, uma alternativa para aumentar a produção de madeira sólida na região Sul da Bahia, para os mais variados fins, seria o plantio das essências florestais no entorno dos SAFs em fileiras simples ou duplas, como também em fileiras simples entre as fileiras duplas de seringueira e nas entrelinhas do cacaueiro. Nesses casos, a colheita seria realizada nas linhas de plantio de espécies madeireiras, evitando danos aos cultivos perenes associados.

\section{Conclusões}

Os resultados mostram que as densidades iniciais de plantio testadas (90 a 130 indivíduos por hectare) não afetaram significativamente o crescimento das árvores. Com isso, conclui-se que densidades iniciais de plantio em torno de 130 árvores por hectare dispensam a necessidade de desbastes intermediários durante a condução das espécies madeireiras e possibilitam uma maior produtividade em volume de madeira. $\mathrm{O}$ alto crescimento das três espécies madeireiras observado neste estudo indica o grande potencial da região Sul da Bahia para produção de madeira sólida, visto que o cultivo de espécies madeireiras em sistemas agroflorestais, além de resultar em uma alta produtividade concentrada em poucas árvores, possibilita ciclos de corte mais curtos e um retorno mais rápido do investimento.

\section{Literatura Citada}

ALMEIDA, L. C. 2001. Principais doenças do cacaueiro e medidas de controle. In: Silva Neto, P. J. (Org.). Sistema de produção de cacau para a Amazônia brasileira, Belém, CEPLAC. pp.63-73.

ASHTON, M. S.; MONTAGNINI, F. 2000. The silvicultural basis for agroforestry systems. CRC Press, Boca Raton, Florida. 296p.

BARTLETT, M. S. 1937. Properties of sufficiency and statistical tests. Proceedings of the Royal Society, Series A, 160:268-282.

CARVAlHO, P. E. R. 2007. Louro-frejó (Cordia alliodora). Circular Técnica 36. Embrapa Florestas. Colombo-PR. 8p.

DA VINHA, S. G.; LOBÃO, D. E. V. P. 1989. Estação Ecológica do Pau-brasil. Centro de Pesquisas do Cacau. 40p.

EVANS, J.; TURNBULL, J. 2004. Plantation Forestry in the Tropics. Oxford University Press, Oxford. 488p.

FAO. 2015. Global forest resources assessment 2015. Food and Agriculture Organization of the United Nations. Rome, Italy. 253p.

FARIA FILHO, A. F.; ARAUJO, Q. R. 2003. Zoneamento do meio físico do Município de Ilhéus, Bahia, Brasil, utilizando a técnica de geoprocessamento. Ilhéus, BA, CEPLAC/ CEPEC. Boletim Técnico no 187. 19p.

IBA. 2016. Indústria Brasileira de Árvores: ano base 2016/IBÁ. Brasília/DF. 100p.

KRISNAWATI, H.; KALLIO, M.; KANNINEN, M. 2011. Swietenia macrophylla King: ecology, 
silviculture and productivity. CIFOR, Bogor, Indonésia. 24p.

LEITE, L. R. C. 2018. Estudo de Competitividade do Cacau e Chocolate no Brasil: Desafios na Produção e Comércio Global. Ministério do Desenvolvimento, Indústria e Comércio Exterior - MDIC. Brasília, DF. 128p.

MARQUES, J. R. B; MONTEIRO, W. R. 2016. Substituição sustentável de eritrina por seringueira em SAF de cacaueiro. Agrotrópica (Brasil) 28(2):101-122.

MAY, H. M.; MOREIRA TROVATTO, C. M. (eds.). 2008. Manual Agroflorestal para a Mata Atlântica. Brasilia, Ministério do Desenvolvimento Agrário, Secretaria de Agricultura Familiar. 196p.

MAYHEW, J. E.; NEWTON, A. C. 1998. The silviculture of mahogany. London, CABI Publishing. 240p.

MONTAGNINI, F. 2017. Integrating landscapes: agroforestry for biodiversity conservation and food sovereignty. Springer. 501p.

NEWBOULD, P. J. 1967. Methods for Estimating the Primary Production of Forest. IBP Handbook 2. Blackwell Scientific Publications, Oxford, UK. 62p.

R DEVELOPMENT CORE TEAM. 2020. R: A language and environment for statistical computing. R Foundation for Statistical Computing, Vienna, Austria.

ROLIM, S. G.; PIOTTO, D. 2018. Silvicultura e
Tecnologia de espécies da Mata Atlântica. Editora Rona, Belo Horizonte. 2018. 160p.

SANTANA, S. O. et al. 2002. Solos da região sudeste da Bahia: atualização da legenda de acordo com o sistema brasileiro de classificação de solos. CEPLAC/EMBRAPA, Rio de Janeiro. 93p.

SCOTT, A. J.; KNOTT, M. 1974. A cluster analysis method for grouping means in the analysis of variance. Biometrics. 30:507-512.

SHAPIRO, S.; WILK, M. 1965. An Analysis of Variance Test for Normality (Complete Samples). Biometrika 52:591-611.

SILVA, I. C. 2013. Sistemas agroflorestais - conceitos e métodos. Itabuna, SBSAF. 308p.

SOMARRIBA, E. et al. 2014. Cocoa-timber agroforestry systems: Theobroma cacao-Cordia alliodora in central America. Agroforestry Systems 88(6):1001-1019.

SOS MATA ATLÂNTICA. 2019. Atlas de Remanescentes da Mata Atlântica. São Paulo: SOS Mata Atlântica/INPE.

UGALDE ARIAS, L. A. 2013. Teak: new trends in silviculture, commercialization, and wood utilization. 1 ed. - International Forestry and Agroforestry, Cartago, Costa Rica. 312p.

ZOMER, R. J. et al. 2016. Global tree cover and biomass carbon on agricultural land: The contribution of agroforestry to global and national carbon budgets. Scientific Reports 6:29987. 